Proc. 45th International Conference on Acoustics, Speech, and Signal Processing

(ICASSP 2020, Barcelona, Spain, May 2020), 2198-2202, 2020.

The final publication is available on IEEE Xplore

\title{
COMPRESSING FLOW FIELDS WITH EDGE-AWARE HOMOGENEOUS DIFFUSION INPAINTING
}

\author{
Ferdinand Jost Pascal Peter Joachim Weickert \\ Mathematical Image Analysis Group, Faculty of Mathematics and Computer Science \\ Campus E1.7, Saarland University, 66041 Saarbrücken, Germany \\ \{jost, peter, weickert\}@mia.uni-saarland.de
}

\begin{abstract}
In spite of the fact that efficient compression methods for dense two-dimensional flow fields would be very useful for modern video codecs, hardly any research has been performed in this area so far. Our paper addresses this problem by proposing the first lossy diffusion-based codec for this purpose. It keeps only a few flow vectors on a coarse grid. Additionally stored edge locations ensure the accurate representation of discontinuities. In the decoding step, the missing information is recovered by homogeneous diffusion inpainting that incorporates the stored edges as reflecting boundary conditions. In spite of the simple nature of this codec, our experiments show that it achieves remarkable quality for compression ratios up to $800: 1$.
\end{abstract}

Index Terms - Flow Fields, Inpainting-based Compression, Homogeneous Diffusion, Discontinuity Preservation

\section{INTRODUCTION}

Flow fields are omnipresent in many applications ranging from scientific visualisation over object tracking to video compression. Most modern video codecs such as HEVC [1] use block matching algorithms to compute motion vectors for coarse blocks of pixels. The resulting piecewise constant motion fields can be encoded very efficiently, but introduce block artefacts. Alternatively, optical flow methods have been used to compute dense flow fields [2, 3] that give a better prediction, but are harder to encode efficiently. Unfortunately, there are no established codecs that specialise on the compression of dense flow fields. Some notable attempts incorporate coding costs into optic flow estimation [4] or come up with an end-to-end framework for video coding with dense flow fields [5]. Another approach focuses on preserving the topology of flow fields for scientific visualisation purposes [6].

This work has received funding from the European Research Council (ERC) under the European Union's Horizon 2020 research and innovation programme (grant agreement no. 741215, ERC Advanced Grant INCOVID).

\subsection{Our Contribution}

The goal of our present paper is to close this gap by proposing a dedicated codec for flow fields. To this end we use ideas from diffusion-based inpainting of sparse data that respects discontinuities. These approaches have shown excellent performance for encoding cartoon-like still images [7] and piecewise smooth depth maps [8]. Although this strategy appears promising since flow fields are often piecewise smooth, it has never been tried before. It requires a number of specific adapations along with a thorough evaluation. We show that a carefully engineered combination of simple ingredients such as Marr-Hildreth edge detection with hysteresis thresholding, homogeneous diffusion inpainting on a sparse regular grid, and uniform quantisation results in a codec that offers excellent performance for compression ratios up to $800: 1$.

\subsection{Related Work}

Compression methods with diffusion-based inpainting were introduced by Galić et al. [9] in 2005. Their method stores only a few selected pixels (so-called mask pixels) of an image and reconstructs the missing data with edge-enhancing anisotropic diffusion (EED) [10]. The R-EED algorithm of Schmaltz et al. [11] improved this idea with a rectangular subdivision that adaptively inserts mask pixels. It can beat the quality of JPEG2000 for some real-world test images.

The concept of diffusion-based inpainting was also extended to video compression. Peter et al. [12] developed a method based on R-EED that allows decoding in real time. However, this approach compresses each frame individually and does not exploit temporal redundancies. Andris et al. [13] proposed a proof-of-concept video codec that additionally uses optical flow methods for inter frame prediction. Their motion fields are compressed with a simple subsampling, again resulting in block artefacts.

The above mentioned techniques were tailored to natural data with a realistic amount of texture. This often requires fairly advanced inpainting methods such as edge-enhancing anisotropic diffusion. However, if one has piecewise smooth data that do not contain texture, simpler operators such as ho- 
mogeneous diffusion inpainting are sufficient, if they are supplemented with additional edge information.

Mainberger et al. [7] developed such a codec for cartoonlike still images. It stores mask pixels on both sides of edges and uses homogeneous diffusion inpainting to reconstruct smooth regions in-between. Gautier et al. [14] have extended these ideas to depth maps. Hoffmann et al. [8] proposed an alternative that explicitly stores segment boundaries, data at a coarse hexagonal grid, and a few additional pixels at locations where the reconstruction quality is poor.

While this discussion shows that homogeneous diffusion inpainting which respects edges is adequate for piecewise smooth still images and depth maps, its usefulness for compressing flow fields is an open problem. This is the topic of our paper. As we will see below, our approach also differs on a technical level from the above mentioned papers: It does not require sophisticated inpainting operators such as in [9.-12], does not use double-sided contour data as in [7, 14], and is substantially simpler than [8].

\subsection{Paper Structure}

In Section 2 we design our edge-aware homogeneous diffusion inpainting operator for flow fields. With this concept we propose the encoding step of our method in Section 3 and the corresponding decoding in Section 4. We evaluate our approach in Section 5 and present our conclusions in Section 6 .

\section{EDGE-AWARE HOMOGENEOUS DIFFUSION INPAINTING}

The centrepiece of our codec is the edge-aware homogeneous diffusion that reconstructs a flow field from a small amount of known data. This method relies on additional edge information to preserve discontinuities of the original flow field. Consider a flow field $\boldsymbol{f}(\boldsymbol{x}): \Omega \rightarrow \mathbb{R}^{2}$ where $\boldsymbol{x}:=(x, y)^{\top}$ denotes the position in a rectangular domain $\Omega \subset \mathbb{R}^{2}$. Flow vectors are only known at mask locations $K \subset \Omega$. We also assume that an edge set $E$ of $\boldsymbol{f}$ is given. The reconstructed flow field $\boldsymbol{u}(\boldsymbol{x})$ can then be computed with homogeneous diffusion inpainting [15] by solving the Laplace equation

$$
\Delta \boldsymbol{u}(\boldsymbol{x})=0 \quad \text { for } \quad \boldsymbol{x} \in \Omega \backslash(K \cap E)
$$

with fixed mask values and reflecting boundary conditions

$$
\begin{aligned}
& \boldsymbol{u}(\boldsymbol{x})=\boldsymbol{f}(\boldsymbol{x}) \quad \text { for } \quad \boldsymbol{x} \in K \\
& \partial_{\boldsymbol{n}} \boldsymbol{u}(\boldsymbol{x})=0 \quad \text { for } \quad \boldsymbol{x} \in \partial \Omega
\end{aligned}
$$

where $\boldsymbol{n}$ denotes the normal vector to the boundary $\partial \Omega$. As an extension to classical homogeneous diffusion we prevent any diffusion across discontinuities by introducing additional reflecting boundary conditions across the boundaries of $E$ :

$$
\partial_{\boldsymbol{n}} \boldsymbol{u}(\boldsymbol{x})=0 \quad \text { for } \quad \boldsymbol{x} \in \partial E
$$

This problem can be discretised with finite differences where edges are given at between-pixel locations. It yields a linear system of equations which we solve with a conjugate gradient method [16]. The result of this edge-aware homogeneous diffusion inpainting gives us a flow field with discontinuities along edges $E$ and smooth transitions in between.

\section{ENCODING}

In this section we describe the encoder of our framework. First it collects edge information at between-pixel locations that act as boundaries for the homogeneous diffusion process. Then it selects the pixel mask needed for inpainting and quantises the selected pixel values. Finally, we use lpaq2 [17] for entropy encoding.

\subsection{Edge Detection}

Our approach uses the Marr-Hildreth operator [18] combined with hysteresis thresholding similar to Canny [19] as an edge detector. It detects zero-crossings of the Laplacian of a Gaussian-smoothed image with a standard deviation of $\sigma$. Additional hysteresis thresholding ensures that we keep only important structures: Edges where the gradient magnitude exceeds a threshold $T_{1}$ become seed points. Then we recursively add all candidates that are adjacent to seed pixels and exceed a threshold $T_{2}<T_{1}$ to the set of edges.

The resulting relevant edges in $x$ - and $y$-direction lie at between-pixel locations on two different grids. This gives us two binary images to encode (Fig. 1). However, following Hoffmann et al. [8], we prefer an alternative that uses chain codes. This is very efficient in our setting as there are only three possible directions to follow. We first extract different types of T-junctions as starting points for our chains (Fig. 2). Additional starting elements (types 5 and 6) are used to cover edges that do not contain any T-junctions. We then follow the contours until there is no more edge to encode. The chain code is a sequence of four different symbols: three symbols for each possible direction and a terminating symbol indicating the end of an edge.

\subsection{Inpainting Mask}

Our edge-aware homogeneous inpainting algorithm also requires a set of mask points to be able to reconstruct a flow field. To reduce the coding cost of our method we choose mask pixels on a regular grid instead of arbitrary positions. Mask positions are uniquely determined by the image dimensions and a density parameter $d$.

Adaptive approaches like the rectangular subdivision of Schmaltz et al. [11] usually give better results for diffusion processes. They accumulate mask pixels around edges to compensate the inability of homogeneous diffusion to preserve edges. Our codec, however, already stores edge infor- 

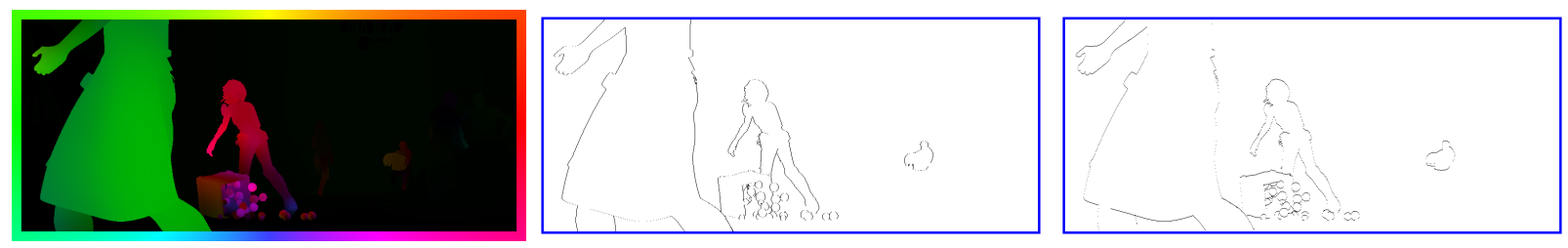

Fig. 1: Edge detection with the Marr-Hildreth edge detector $\left(\sigma=0.5, T_{1}=4, T_{2}=2\right)$. Left: Colour-coded original flow field. Middle: Edges in $x$-direction. Right: Edges in $y$-direction.

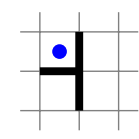

type 1

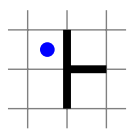

type 2

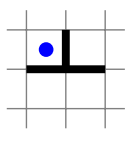

type 3

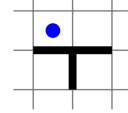

type 4

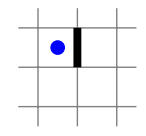

type 5

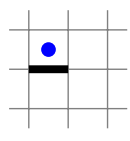

type 6

Fig. 2: Types of T-junctions with reference points in blue.

mation explicitly. This way adaptive schemes do not provide a large benefit over a regular mask. In this case the small improvement in quality that these methods provide cannot compensate for the overhead of storing a more complex mask.

As edges can form isolated segments that do not contain a point of the regular grid, we additionally store the average flow value of such segments. For homogeneous diffusion, this is equivalent to storing a single mask pixel at an arbitrary position inside this segment.

\subsection{Quantisation}

As flow vectors usually consist of two 32-bit floating-point numbers, we want to represent the range of actually occurring values as integers $q \in\{0, \ldots, k-1\}$. One of the simplest methods to achieve this is uniform quantisation.

Let $\min$ be the minimal and $\max$ the maximal value occurring in one channel of the flow field, and let $x \in$ $[\min , \max ]$ be a flow value. We also define the length of a quantisation interval as $a:=\frac{\max -\min }{k-1}$. The quantised value $q$ can then be computed as $q=\left\lfloor\frac{x-\text { min }}{a}+\frac{1}{2}\right\rfloor$. In order to reconstruct a flow value $x_{q}$ we can simply compute $x_{q}=\min +a \cdot q$. The first and last interval only have a width of $\frac{a}{2}$. This preserves the minimal and maximal flow values.

\section{DECODING}

The decoding step of our codec is a straightforward process. We first reconstruct the regular inpainting mask using the stored density parameter $d$. Next we follow the chain codes starting at T-junctions in each direction until we encounter a terminating symbol to reconstruct edges. Finally, we restore flow vectors from the stored quantised values and place them on their corresponding mask positions. The flow field is then reconstructed by solving the edge-aware homogeneous diffusion inpainting problem proposed in Section 2

\section{EXPERIMENTS}

In this section we demonstrate the potential of our framework using two ground truth flow fields of the MPI Sintel Flow Dataset [20] as test images. This provides us with high quality flow fields with realistic complexity. Since there are no standards for the compression of flow fields, we compare our method to JPEG2000 [21] and BPG [22], an adaption of HEVC's intra-coding mode for still images [23]. Those are the best established alternatives for arbitrary images, but are not designed to handle floating-point pixel data. Therefore, we quantise the input flow fields channelwise to integer values in the range $\{0, \ldots, 255\}$ with the uniform quantisation described in Section 3. We also use these quantised flow fields as inputs to our method. To optimise the parameters of our approach we perform a simple grid search. We fix the standard deviation $\sigma$ of the edge detection to 0.5 , which performs well in our experiments.

Fig. 3 shows the results of JPEG2000, BPG, and our edgeaware approach for a compression ratio of $400: 1$. The compression ratio is computed relative to the quantised input flow fields. We observe that transform-based codecs like JPEG2000 have problems preserving sharp edges. This results in unpleasant ringing artefacts around object boundaries. BPG performs much better than JPEG2000, but still blurs edges. In contrast, our edge-aware homogeneous diffusion inpainting reconstructs all important edges very well. In a quantitative comparison we measure the peak-signal-to-noise ratio (PSNR) of the different approaches. Fig. 4 shows that our method outperforms both JPEG2000 and BPG for all measured compression ratios, ranging from $10: 1$ to $800: 1$.

\section{CONCLUSIONS AND OUTLOOK}

In our paper, we have presented the first diffusion-based compression approach for dense flow fields. We have shown that a careful combination of very simple concepts ranging from Marr-Hildreth edge detection to uniform quantisation is already sufficent to achieve a remarkable quality even at very high compression ratios. Our proof-of-concept framework is very flexible: Its modular nature allows to adapt and improve it by simply exchanging the different components. In our ongoing work, we are investigating this option further. We will also embed our approach into existing video codecs. 

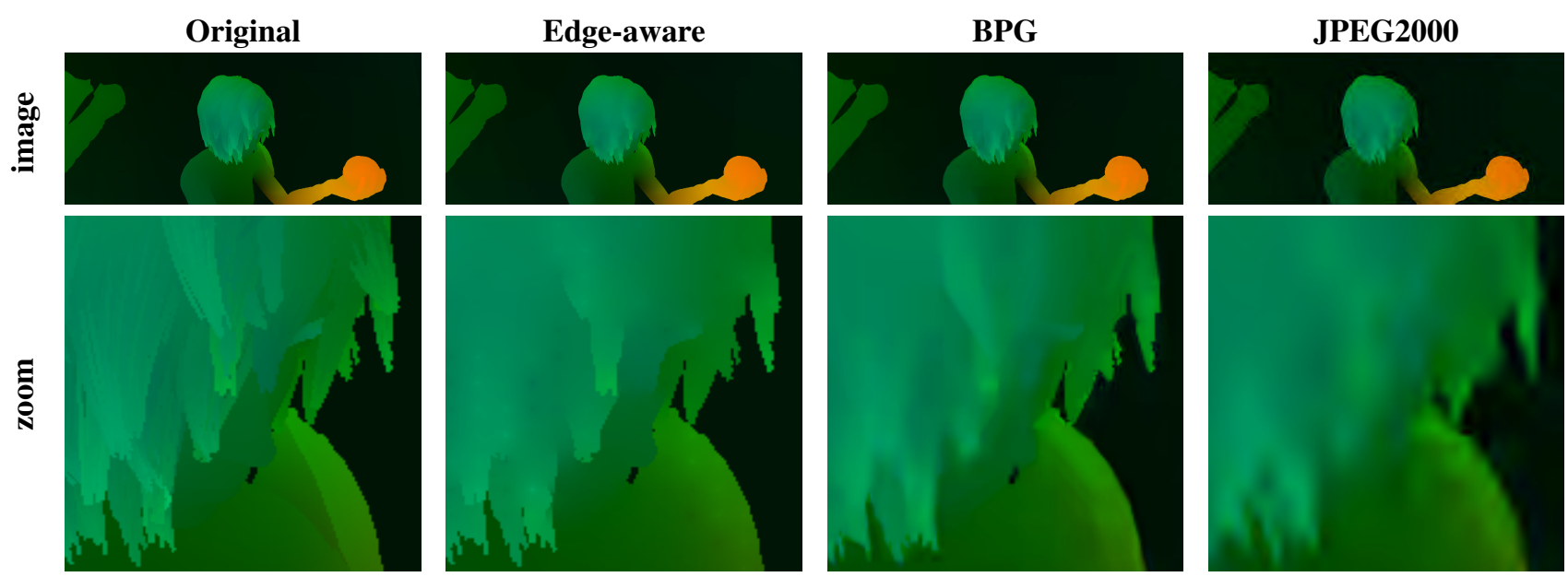

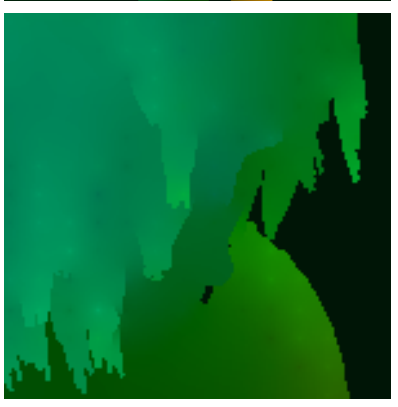

PSNR: 46.10
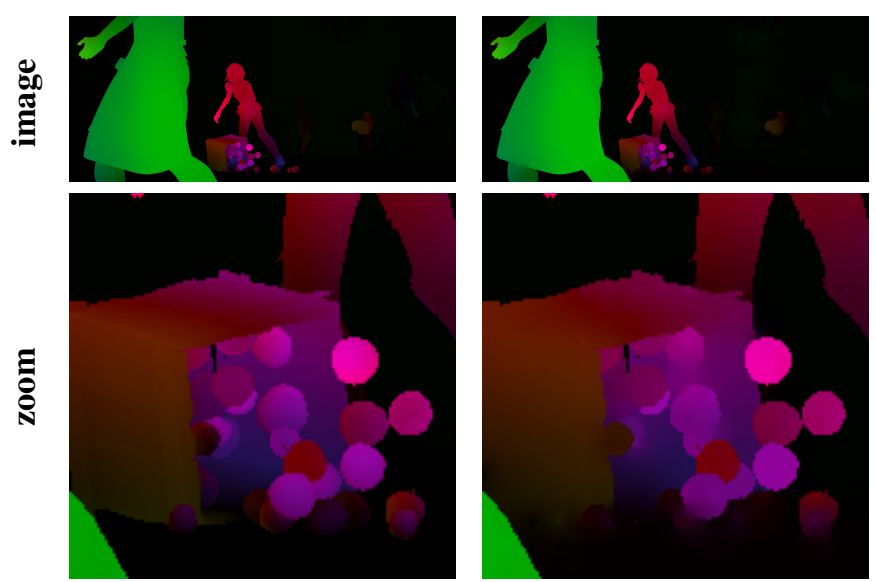

PSNR: 44.20

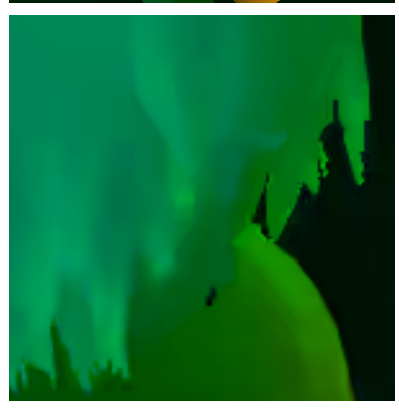

PSNR: 41.73
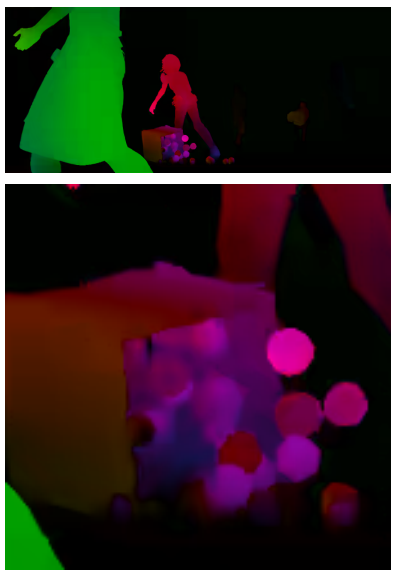

PSNR: 40.65

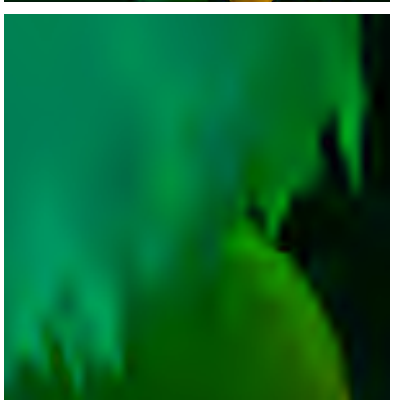

PSNR: 36.47
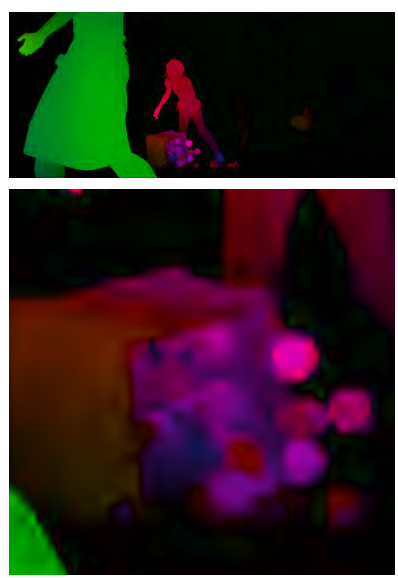

PSNR: 36.42

Fig. 3: Comparison of JPEG2000, BPG, and our edge-aware approach for two flow fields at a compression ratio of 400 : 1. Both JPEG2000 and BPG show artefacts around edges, whereas our edge-aware approach preserves edges more accurately.
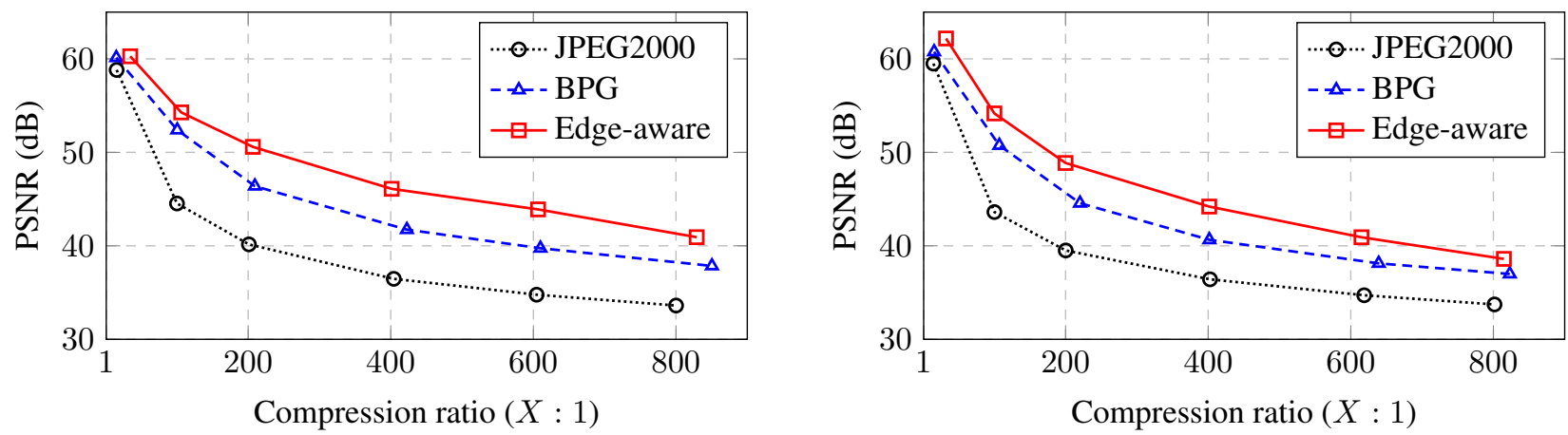

Fig. 4: Comparison of our method with JPEG2000 and BPG in terms of PSNR (higher is better). Our codec outperforms JPEG2000 and BPG consistently on both alley (left) and market (right).

\section{REFERENCES}

[1] Moving Pictures Experts Group and Video Coding Experts Group, "Information technology - High efficiency coding and media delivery in heterogeneous environments - Part 2: High efficiency video coding," ISO/IEC 23008-2:2017. 
[2] R. Krishnamurthy, P. Moulin, and J. W. Woods, "Optical flow techniques applied to video coding," in Proc. 1995 International Conference on Image Processing, Washington, DC, Oct. 1995, pp. 570-573, IEEE Computer Society.

[3] S.-C. Han and C. Podilchuk, "Video compression with dense motion fields," IEEE Transactions on Image Processing, vol. 10, no. 11, pp. 1605-1612, Nov. 2001.

[4] G. Ottaviano and P. Kohli, "Compressible motion fields," in 2013 IEEE Conference on Computer Vision and Pattern Recognition, Portland, OR, June 2013, pp. 2251-2258, IEEE Computer Society.

[5] G. Lu, W. Ouyang, D. Xu, X. Zhang, C. Cai, and Z. Gao, "DVC: An end-to-end deep video compression framework," in 2019 IEEE Conference on Computer Vision and Pattern Recognition, Long Beach, CA, June 2019, pp. 11006-11015, IEEE Computer Society.

[6] H. Theisel, C. Rössl, and H.-P. Seidel, "Compression of $2 \mathrm{D}$ vector fields under guaranteed topology preservation," Computer Graphics Forum, vol. 22, no. 3, pp. 333-342, 2003.

[7] M. Mainberger, A. Bruhn, J. Weickert, and S. Forchhammer, "Edge-based compression of cartoon-like images with homogeneous diffusion," Pattern Recognition, vol. 44, no. 9, pp. 1859-1873, Sept. 2011.

[8] S. Hoffmann, M. Mainberger, J. Weickert, and M. Puhl, "Compression of depth maps with segment-based homogeneous diffusion," in Scale Space and Variational Methods in Computer Vision, A. Kuijper, K. Bredies, T. Pock, and H. Bischof, Eds., vol. 7893 of Lecture Notes in Computer Science, pp. 319-330. Springer, Berlin, 2013.

[9] I. Galić, J. Weickert, M. Welk, A. Bruhn, A. Belyaev, and H.-P. Seidel, "Towards PDE-based image compression," in Variational, Geometric and Level-Set Methods in Computer Vision, N. Paragios, O. Faugeras, T. Chan, and C. Schnörr, Eds., vol. 3752 of Lecture Notes in Computer Science, pp. 37-48. Springer, Berlin, 2005.

[10] J. Weickert, "Theoretical foundations of anisotropic diffusion in image processing," Computing Supplement, vol. 11, pp. 221-236, 1996.

[11] C. Schmaltz, P. Peter, M. Mainberger, F. Ebel, J. Weickert, and A. Bruhn, "Understanding, optimising, and extending data compression with anisotropic diffusion," International Journal of Computer Vision, vol. 108, no. 3, pp. 222-240, July 2014.
[12] P. Peter, C. Schmaltz, N. Mach, M. Mainberger, and J. Weickert, "Beyond pure quality: Progressive mode, region of interest coding and real time video decoding in PDE-based image compression," Journal of Visual Communication and Image Representation, vol. 31, pp. 256-265, Aug. 2015.

[13] S. Andris, P. Peter, and J. Weickert, "A proof-ofconcept framework for PDE-based video compression," in Proc. 32nd Picture Coding Symposium, Nuremberg, Germany, Dec. 2016.

[14] J. Gautier, O. Le Meur, and C. Guillemot, "Efficient depth map compression based on lossless edge coding and diffusion," in Proc. 2012 Picture Coding Symposium, Kraków, Poland, May 2012, pp. 81-84.

[15] T. Iijima, "Basic theory on normalization of pattern (in case of typical one-dimensional pattern)," Bulletin of the Electrotechnical Laboratory, vol. 26, pp. 368-388, 1962, In Japanese.

[16] Y. Saad, Iterative Methods for Sparse Linear Systems, SIAM, Philadelphia, second edition, 2003.

[17] M. Mahoney, "Adaptive weighing of context models for lossless data compression," Tech. Rep. CS-200516, Florida Institute of Technology, Melbourne, Florida, Dec. 2005.

[18] D. Marr and E. Hildreth, "Theory of edge detection," Proceedings of the Royal Society of London, Series B, vol. 207, pp. 187-217, 1980.

[19] J. Canny, "A computational approach to edge detection," IEEE Transactions on Pattern Analysis and Machine Intelligence, vol. 8, pp. 679-698, 1986.

[20] D. J. Butler, J. Wulff, G. B. Stanley, and M. J. Black, "A naturalistic open source movie for optical flow evaluation," in Computer Vision - ECCV 2012, Part IV, A. W. Fitzgibbon, S. Lazebnik, P. Perona, Y. Sato, and C. Schmid, Eds., vol. 7577 of Lecture Notes in Computer Science, pp. 611-625. Springer, Berlin, 2012.

[21] D. S. Taubman and M. W. Marcellin, Eds., JPEG 2000: Image Compression Fundamentals, Standards and Practice, Kluwer, Boston, 2002.

[22] F. Bellard, "Better portable graphics (BPG)," https://bellard.org/bpg/.

[23] T. Nguyen and D. Marpe, "Performance analysis of HEVC-based intra coding for still image compression," in Picture Coding Symposium, PCS 2012, M. Domanski, T. Grajek, D. Karwowski, and R. Stasinski, Eds., Krakow, Poland, May 2012, pp. 233-236. 